
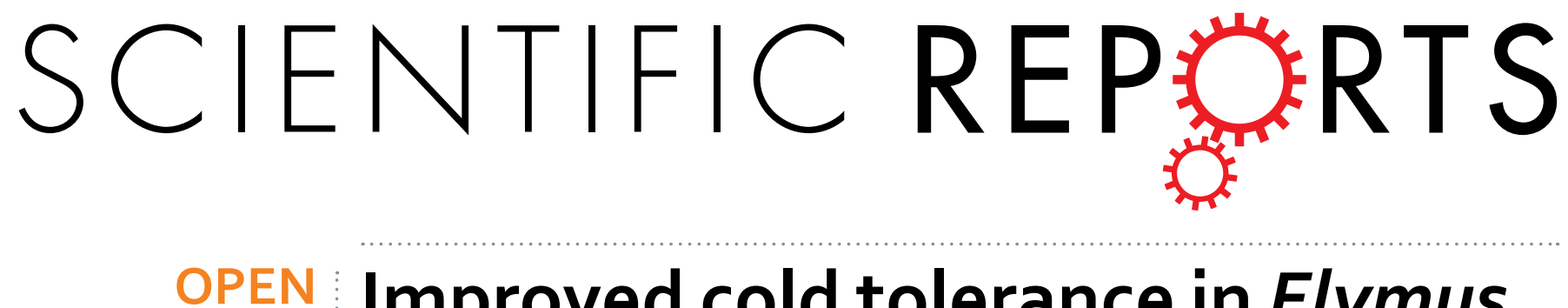

\title{
Improved cold tolerance in Elymus nutans by exogenous application of melatonin may involve ABA-
}

Received: 14 July 2016

Accepted: 28 November 2016

Published: 03 January 2017 dependent and $A B A$-independent pathways

Juanjuan Fu ${ }^{1}$, Ye Wu ${ }^{1}$, Yanjun Miao ${ }^{2}$, Yamei Xu ${ }^{2}$, Enhua Zhao ${ }^{1}$, Jin Wang ${ }^{1}$, Huaien Sun ${ }^{1}$, Qian Liu ${ }^{1}$, Yongwei Xue ${ }^{3}$, Yuefei $\mathrm{Xu}^{1}$ \& Tianming Hu${ }^{1}$

Melatonin is an important secondary messenger that plays a central role in plant growth, as well as abiotic and biotic stress tolerance. However, the underlying physiological and molecular mechanisms of melatonin-mediated cold tolerance, especially interactions between melatonin and other key molecules in the plant stress response, remain unknown. Here, the interrelation between melatonin and abscisic acid (ABA) was investigated in two genotypes of Elymus nutans Griseb., the coldtolerant Damxung (DX) and the cold-sensitive Gannan (GN) under cold stress. Pre-treatment with exogenous melatonin or $A B A$ alleviated oxidative injury via scavenging $R O S$, while enhancing both antioxidant enzyme activities and non-enzymatic antioxidant contents. Treatment of fluridone, an ABA biosynthesis inhibitor caused membrane lipid peroxidation and lowered melatonin-induced antioxidant defense responses. It is worth noting that cold stress significantly induced both endogenous melatonin and $A B A$ levels in both genotypes. Application of melatonin increased $A B A$ production, while fluridone significantly suppressed melatonin-induced $A B A$ accumulation. $A B A$ and fluridone pre-treatments failed to affect the endogenous melatonin concentration. Moreover, exogenous melatonin up-regulated the expression of cold-responsive genes in an ABA-independent manner. These results indicate that both $A B A$-dependent and $A B A$-independent pathways may contribute to melatonin-induced cold tolerance in E. nutans.

Cold stress presents one of the major limitations for plant growth and yield worldwide, especially in areas of high altitude due to its negative effects on plant physiology, biochemistry, and molecular biology ${ }^{1}$. Changes in membrane fluidity and composition under cold exposure trigger the accumulation of various osmoprotectants, thus alleviating oxidative damage ${ }^{2,3}$. At molecular level, low but non-freezing temperatures have been reported to induce rapid expression of transcription factors and cold-regulated genes, resulting in an enhanced freezing tolerance ${ }^{4}$. This complex response is comprised of two major pathways and which one will be utilized depends on the involvement of abscisic acid $(\mathrm{ABA})^{5}$. One of the known major ABA-independent cold-signaling pathways is the ICE (inducer of CBF expression)-CBF (C-repeat binding factor)-COR (cold regulated genes) transcriptional cascade $^{6}$. In cereals, $C B F 14$ was found to have maximal effect on freezing tolerance ${ }^{7-9}$. One of the first detectable gene expressions induced by low temperatures was the rapid and controlled induction of $C B F$ genes, increasing simultaneously cold tolerance ${ }^{7}$. The $C B F$ response is furthermore followed by the expression of the COR gene $e^{10}$. In Triticeae, COR expression levels are correlated with freezing tolerance: genotypes with better freezing tolerance accumulate COR gene transcripts in higher amounts compared to genotypes with lower freezing tolerance ${ }^{11}$. The

${ }^{1}$ Department of grassland science, College of Animal Science and Technology, Northwest A\&F University, Yangling, Shaanxi, 712100, China. ${ }^{2}$ College of Plant Science, Tibet Agriculture and Animal Husbandry College, Linzhi, Tibet, 860000 , China. ${ }^{3}$ Department of grassland ecology, College of Desertification Prevention Engineering, Ningxia Technical College of Wine and Desertification Prevention, Yongning, Yinchuan, 750001, China. Correspondence and requests for materials should be addressed toY.X. (email: xuyuefei1980@163.com) orT.H. (email: hutianming@126. com) 
second pathway participating in the cold acclimation process is ABA-dependent and is induced via dehydration instead of the lowered temperature itself. This response is slow and it includes bZIP transcription factors known as ABA Responsive Element Binding Protein/Factors $(\mathrm{AREB} / \mathrm{ABF})^{12}$. Both pathways are not independent, but are linked via complex interrelations ${ }^{13,14}$.

Hormone treatment has been utilized as an approach to alleviate various abiotic and biotic stresses in plants ${ }^{15}$. Melatonin (N-acetyl-5-methoxytryptamine) is an important animal hormone that has been reported to be involved in multiple biological processes ${ }^{16}$. Although its function as a hormone has been well established in animals, functional knowledge in higher plant is still very limited. In recent years, melatonin has been found to be a ubiquitous modulator in multiple plant developmental processes, including flowering, promotion of photosynthesis, preservation of chlorophyll ${ }^{17}$, stimulation and regeneration of root system architecture ${ }^{18}$, delayed senescence of leaves ${ }^{19}$, and alleviation of oxidative damage induced by reactive oxygen species (ROS) and reactive nitrogen species $(\mathrm{RNS})^{17,20}$. Moreover, melatonin is involved in the regulation of various abiotic stresses, such as cold ${ }^{21-23}$, salinity ${ }^{24}$, heavy metal ${ }^{25}$, herbicides ${ }^{26}$, and UV radiation ${ }^{27}$. The mechanisms of melatonin-mediated stress tolerance involve the activation of antioxidants biosynthesis and activities of antioxidant enzymes, as well as the direct scavenging of ROS following plant exposure to harsh environments ${ }^{28-30}$. Despite melatonin positively mediating plant responses to cold stress (e.g. via promoting seed germination in cucumber ${ }^{28}$, mitigating oxidative damage in maize seedlings $\mathrm{s}^{23}$, and up-regulating the expression of cold-induced transcriptional activators including CBFs and zinc finger of Arabidopsis thaliana 6 (ZAT6) ${ }^{30,31}$ ), the exact mechanism enabling these responses remain largely unknown.

The plant hormone ABA acts as a stress signal in plants and plays an important role in modulating plant response to various biotic and abiotic stresses, including cold stress ${ }^{32}$. A previous study showed that exogenous ABA application before the onset of cold stress improved cold resistance of plants ${ }^{33}$. ABA can improve the antioxidant defense system, thus protecting plant cells from damage caused by over-accumulated ROS $^{34}$. Accumulating evidence has shown that ABA interacts with other important signaling molecules, such as nitric oxide (NO), hydrogen peroxide $\left(\mathrm{H}_{2} \mathrm{O}_{2}\right)$, and calcium $\left(\mathrm{Ca}^{2+}\right)$, thus participating in the regulation of cold-tolerance responses $^{35,36}$. However, the interrelation between melatonin and ABA in the acquisition of cold tolerance remains unclear.

To improve our understanding of melatonin function and its potential interrelation with ABA in plants exposed to cold conditions, two genotypes of Elymus nutans Griseb., the cold-tolerant Damxung (DX) and the cold-sensitive Gannan (GN) were studied. E. nutans is a perennial Triticeae cool-season grass, with a distribution in the northern, northwestern, and southwestern regions of China. It is especially prevalent on the alpine meadow of the Qinghai-Tibetan Plateau where temperatures greatly fluctuate ${ }^{37}$. Thus, these grasses have evolved specific physiological mechanisms to adapt to changing environmental conditions. A detailed analysis of the cold adaptation of $E$. nutans will expand our understanding of cold tolerance in plants in general. In this study, the endogenous melatonin levels and cold-responsive genes were examined and quantified in both genotypes when exposed to cold stress. Our data revealed that endogenously produced melatonin and expressions of EnCBFs and EnCOR14a genes were significantly increased due to cold stress in both genotypes. Based on pharmacological and biochemical analyses, the present study suggests that melatonin-induced cold tolerance in $E$. nutans works via both ABA-dependent and ABA-independent pathways.

\section{Results}

Exogenous melatonin and ABA alleviates cold stress-induced growth inhibition and cell membrane damage. Cold stress resulted in significant growth suppression in two E. nutans seedlings, with fresh weights decreasing by $39.4 \%$ (GN) and $32.1 \%$ (DX) compared to the control during the $120 \mathrm{~h}$ of cold treatment (Fig. 1a,c). The relative electrolyte leakage level was increased $(P<0.05)$ in both genotypes under cold stress, and values were lower in DX than in GN (Fig. 1b,d).

To determine the appropriate level of melatonin for plant growth, fresh weight of the two E. nutans genotypes were compared following cultivation on various melatonin levels $(0-300 \mu \mathrm{M})$. Our results revealed that the best growth and development could be obtained with melatonin concentrations ranging from 10 to $50 \mu \mathrm{M}$ after $120 \mathrm{~h}$ of cold stress, whereas higher melatonin doses $(300 \mu \mathrm{M})$ had no protective effect on $(P<0.05)$ plant growth (Fig. 1a). Exogenous melatonin treatment had no significant effect on shoot length and fresh weight under normal conditions (Supplementary Table S1). Similarly, melatonin affected electrolyte leakage in a dosage-dependent manner when plants were subjected to cold stress. While melatonin concentrations of $10-100 \mu \mathrm{M}$ reduced cold stress-induced electrolyte leakage, reaching an optimal dose at $50 \mu \mathrm{M}$ melatonin treatment, higher concentrations of melatonin $(300 \mu \mathrm{M})$ resulted in no protective effect on membranes (Fig. 1b). In summary, 50 $\mu \mathrm{M}$ melatonin was most effective to improve the resistance to cold-induced membrane damage for both tested genotypes.

Pre-treatment with exogenous ABA was performed at concentrations of $0,5,10,50,100$, and $200 \mu \mathrm{M}$ to determine the concentration that leads to the most significant effect on cold stress tolerance. The results show that ABA concentrations from 50 to $100 \mu \mathrm{M}$ alleviated the loss of fresh weight as well as the electrolyte leakage level, with $100 \mu \mathrm{M}$ being most effective in the leaves of DX and GN under cold stress (Fig. 1c,d). Since $50 \mu \mathrm{M}$ melatonin and $100 \mu \mathrm{M} \mathrm{ABA}$ appeared to be the most effective in enhancing cold tolerance in E. nutans, these concentrations were used in all subsequent experiments.

ABA is involved in melatonin-induced reduction in oxidative damage. To investigate the role of ABA in melatonin alleviated oxidative damage caused by cold stress, an inhibitor of endogenous ABA biosynthesis was used (fluridone). The electrolyte leakage, MDA concentrations, and ROS levels for each E. nutans genotypes were increased $(P<0.05)$ after $120 \mathrm{~h}$ of cold stress and values were lower in the cold-tolerant DX than in the cold-sensitive GN (Fig. 2a-d). However, exogenous application of $50 \mu \mathrm{M}$ melatonin or $100 \mu \mathrm{M}$ ABA to the roots via irrigation solution alleviated $(P<0.05)$ this response over the course of the stress period. The beneficial effects 
(a)

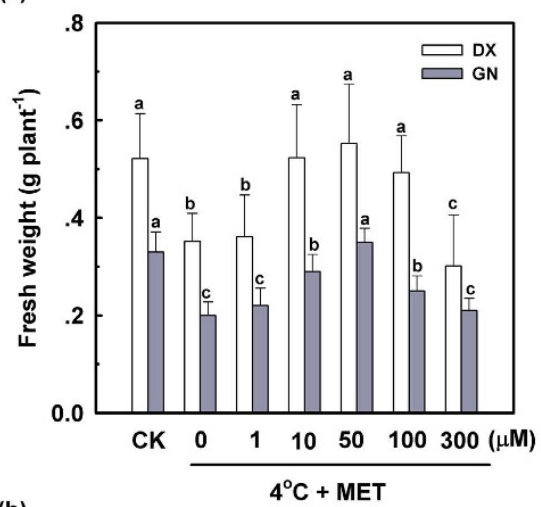

(b)

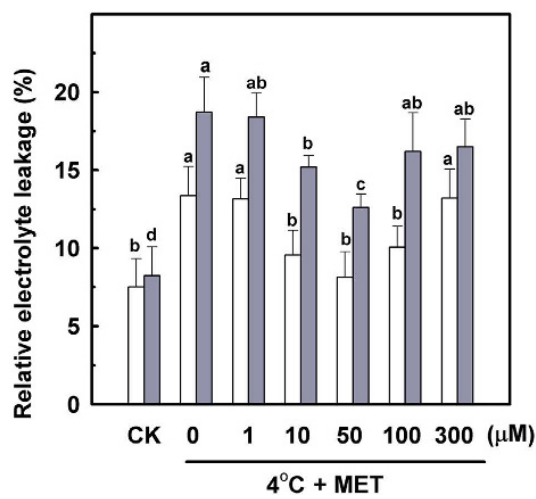

(c)

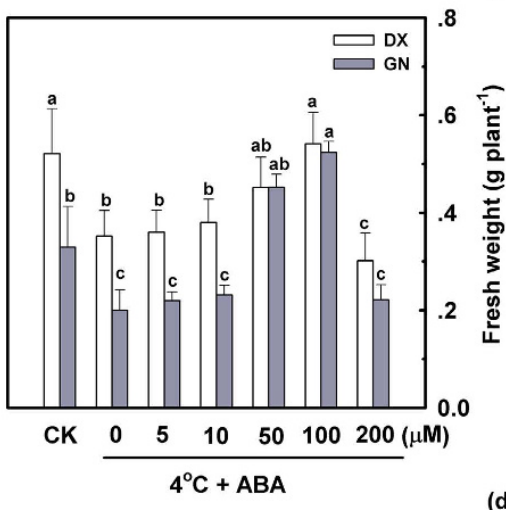

(d)

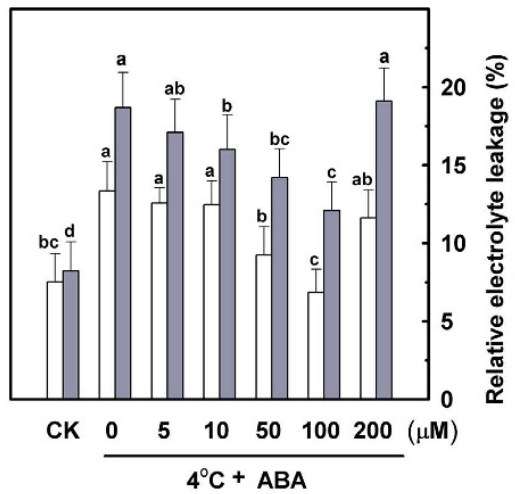

Figure 1. Effects of different melatonin and $A B A$ concentrations on fresh weight $(\mathbf{a}, \mathbf{c})$ and electrolyte leakage $(\mathbf{b}, \mathbf{d})$ in DX and GN seedlings under cold stress $\left(4^{\circ} \mathrm{C}\right)$. Plants treated with distilled water under the normal conditions $\left(25^{\circ} \mathrm{C}\right)$ served as controls. Each value represents the mean of three replicates $\pm \mathrm{SD}$, and different letters above the bars indicate significant differences at $P<0.05$ among different treatments in the same genotype according to Duncan's multiple range test.

of exogenous melatonin and ABA were more profound for the cold-sensitive genotype than for cold-tolerant plants, as indicated by melatonin-induced declines in electrolyte leakage, MDA concentration, $\mathrm{H}_{2} \mathrm{O}_{2}$ accumulation, and superoxide radical production (by $26 \%, 42.2 \%, 32.7 \%$, and $37.1 \%$ (tolerant) vs $33.2 \%, 58.1 \%, 42.5 \%$, and $47.7 \%$ (sensitive), respectively). Pre-treatment with fluridone under simultaneous presence of melatonin evidently increased electrolyte leakage, MDA concentration, and ROS levels. However, these treatments alone failed to affect membrane lipid peroxide levels and ROS concentrations in control leaves (Supplementary Table S2). These results revealed ABA involvement in exogenous melatonin-induced reduction in membrane lipid peroxide levels and ROS concentrations.

ABA is required for exogenous melatonin-induced antioxidant defense. Cold stress caused an increase in non-enzymatic antioxidant concentrations and in the activities of antioxidant enzymes in leaves from all sampled plants. Values were further increased via application of melatonin and ABA. An exogenously applied $50 \mu \mathrm{M}$ of fluridone led to a decline in concentrations of GSH, AsA, total glutathione, and total ascorbate after $120 \mathrm{~h}$ of cold stress. Combining melatonin and fluridone declined $(P<0.05)$ the concentrations of these antioxidants (Fig. 3a-d). Activities of SOD, CAT, APX, and GR (Fig. 4a-d) increased due to melatonin exposure under cold stress, especially in GN leaves. However, pre-treatment with fluridone alone did not affect the concentrations of non-enzymatic antioxidants or the activities of antioxidant enzymes in the control leaves (Supplementary Tables S3 and S4). These results suggest that ABA is required for melatonin-induced stimulation of antioxidant defense and alleviating oxidative damage in cold-stressed $E$. nutans leaves.

Endogenous melatonin and ABA concentrations in response to cold stress. To reveal the relationship between cold stress and melatonin concentration, the endogenous melatonin levels in leaves of DX and GN were quantified after exposure to cold stress $\left(4^{\circ} \mathrm{C}\right)$ for $0,1,3,6,12,24$, and $120 \mathrm{~h}$. Endogenous melatonin contents of both genotypes were approximately $45 \mathrm{pg} \mathrm{g}^{-1} \mathrm{FW}$ before transfer to cold conditions. When exposed to cold stress $\left(4^{\circ} \mathrm{C}\right)$, the endogenous melatonin content in both genotypes continuously increased compared to plants cultured in control conditions within $120 \mathrm{~h}$ cold stress (Fig. 5a,b). Melatonin-treated plants showed a further increase in endogenous melatonin content compared to non-treated plants during the whole cold stress period. This was even more marked in sensitive species, demonstrating that those plants could not produce melatonin as efficiently as plants with improved cold tolerance. This significant induction of the endogenous melatonin concentration via cold stress indicated a direct involvement of melatonin in the E. Nutans response to cold 
(a)

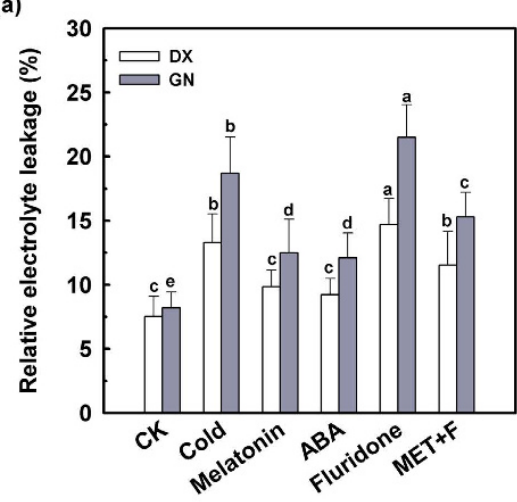

(c)

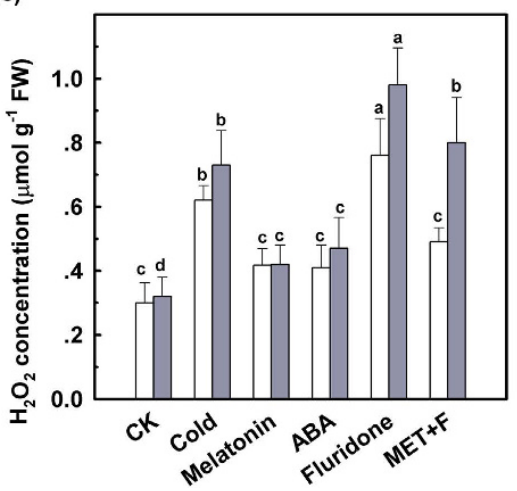

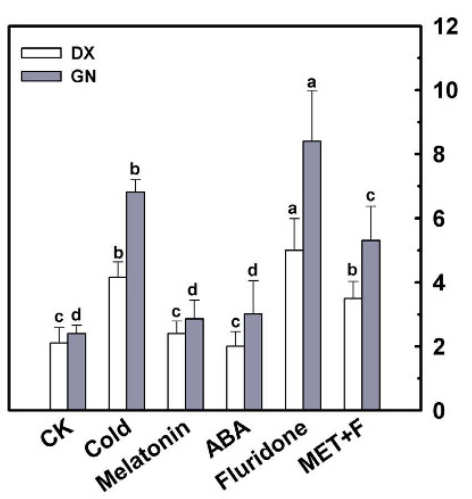

(b)

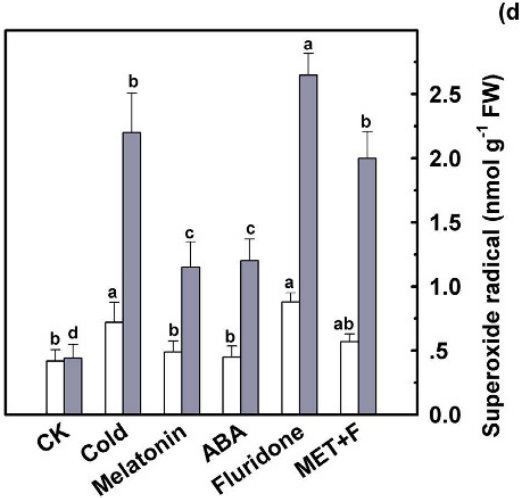

Figure 2. Effect of melatonin and ABA on electrolyte leakage (a), MDA content (b), $\mathrm{H}_{2} \mathrm{O}_{2}$ (c) and superoxide radical (d) accumulation in leaves of DX and GN under cold stress. The 21-day-old seedlings were pretreated with distilled water, $50 \mu \mathrm{M}$ melatonin, $100 \mu \mathrm{M}$ ABA, $50 \mu \mathrm{M}$ fluridone, and $50 \mu \mathrm{M}$ melatonin $+50 \mu \mathrm{M}$ fluridone $(\mathrm{MET}+\mathrm{F})$ for 7 days, respectively, and then exposed to cold stress $\left(4^{\circ} \mathrm{C}\right)$ for $120 \mathrm{~h}$. Plants treated with distilled water under the normal conditions $\left(25^{\circ} \mathrm{C}\right)$ served as controls. Each value represents the mean of three replicates $\pm \mathrm{SD}$, and different letters above the bars indicate significant differences at $P<0.05$ among different treatments in the same genotype according to Duncan's multiple range test.

stress. Accordingly, the endogenous ABA levels in both species immediately increased when subjected to $1 \mathrm{~h}$ of cold stress, reached their maximal level at $3 \mathrm{~h}$, and remained at similar levels from $6 \mathrm{~h}$ to $120 \mathrm{~h}$. Notably, increased endogenous ABA levels were observed in DX compared to GN after $3 \mathrm{~h}$ of cold stress, lasting until the experiment ended. A further increase of endogenous ABA concentration was observed after exogenous melatonin treatment, while the increases were blocked $(P<0.05)$ by pre-treatment with the ABA biosynthesis inhibitor fluridone in both plants during the cold treatment period (Fig. $5 \mathrm{c}, \mathrm{d}$ ). The inhibitory effects were more pronounced for cold-sensitive GN than for the cold-tolerant DX. Fluridone treatment had no significant effect on ABA concentration in both E. nutans genotypes grown in normal conditions (Supplementary Table S5). Pre-treatment with exogenous melatonin and melatonin + fluridone $(\mathrm{MET}+\mathrm{F})$ increased $(P<0.05)$ endogenous melatonin contents in leaves of DX and GN throughout the treatment. However, application of ABA or fluridone alone failed to affect the endogenous melatonin production in both genotypes compared to non-treated plants under cold stress. This indicates that melatonin-induced cold signaling pathway might be ABA-independent.

Effect of melatonin and ABA on cold-regulated genes. Cold stress has been reported to induce the expression of the CBF family of transcription fators, which in turn activate a set of downstream COR genes protecting plants from cold-induced injury ${ }^{4,5}$. According to previous transcriptome data, the levels of EnCBF9, $E n C B F 14$, and $E n C O R 14 a$ were significantly induced due to cold treatment in both $E$. nutans genotypes (Supplementary Table S6). As shown in Fig. 6, the expressions of EnCBF9 and EnCBF14 were immediately induced in both genotypes exposed to cold treatment at $1 \mathrm{~h}$ and $3 \mathrm{~h}$, and reached a maximal value at $3 \mathrm{~h}$ and $6 \mathrm{~h}$, followed by a decline to levels still well above those prior to cold exposure. Compared to non-melatonin-treated plants under cold stress, both genotypes treated with exogenous melatonin had higher $(P<0.05)$ levels of EnCBF9 and $E n C B F 14$ transcripts during cold stress. The expression levels of EnCBF9 immediately increased within $1 \mathrm{~h}$ of exposure to cold stress and melatonin treatment, and decreased until $12 \mathrm{~h}$, while increasing at $24 \mathrm{~h}$. After $120 \mathrm{~h}$ of cold treatment, the expression level of EnCBF9 declined in the melatonin-treated plants, but significantly increased $(P<0.05)$ compared to non-melatonin-treated plants under cold stress. The transcript of EnCBF14 genes in GN genotypes immediately increased within $1 \mathrm{~h}$ of exposure to cold stress and melatonin treatment, peaked at $3 \mathrm{~h}$, and subsequently declined until $120 \mathrm{~h}$ of cold treatment. Pre-treatment with ABA and fluridone had no significant inhibitory effect on the expression of the two EnCBFs in cold treated E. nutans. 
(a)

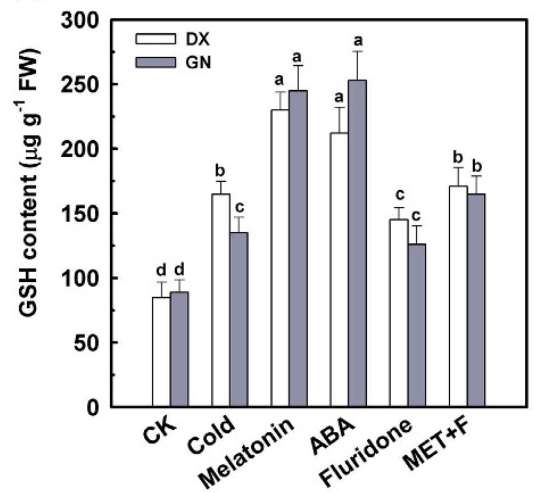

(c)

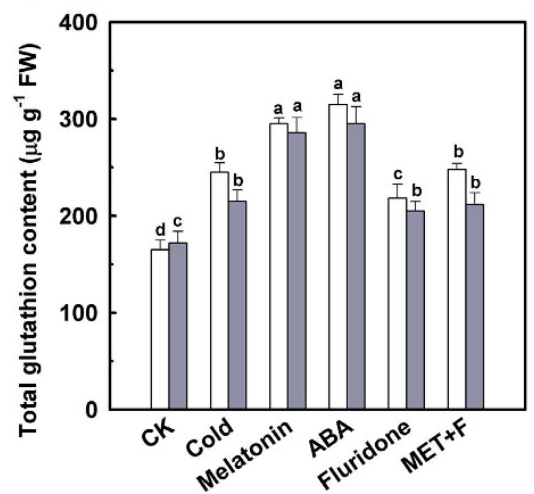

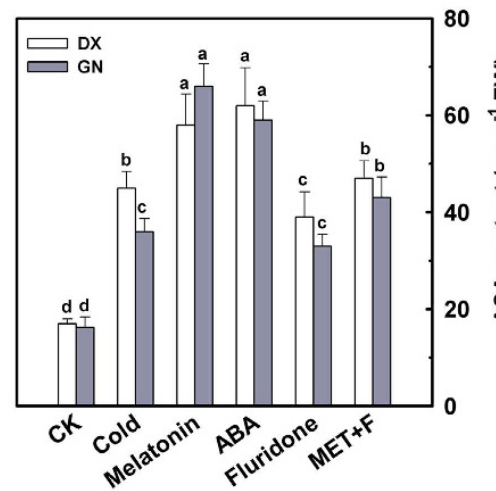

(b)

80

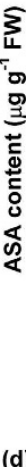

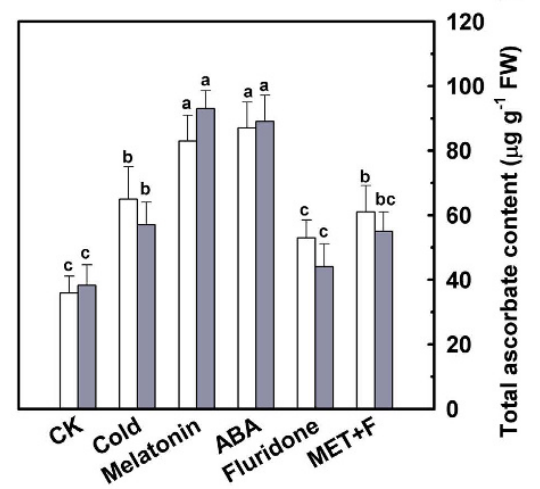

Figure 3. Effect of melatonin and ABA on contents of non- enzymatic antioxidants GSH (a), AsA (b), Total glutathione (c) and ascorbate (d) in leaves of DX and GN under cold stress. The 21-day-old seedlings were pretreated with distilled water, $50 \mu \mathrm{M}$ melatonin, $100 \mu \mathrm{M} \mathrm{ABA}, 50 \mu \mathrm{M}$ fluridone, and $50 \mu \mathrm{M}$ melatonin $+50 \mu \mathrm{M}$ fluridone $(\mathrm{MET}+\mathrm{F})$ for 7 days, respectively, and then exposed to cold stress $\left(4^{\circ} \mathrm{C}\right)$ for $120 \mathrm{~h}$. Plants treated with distilled water under the normal conditions $\left(25^{\circ} \mathrm{C}\right)$ served as controls. Each value represents the mean of three replicates $\pm \mathrm{SD}$, and different letters above the bars indicate significant differences at $P<0.05$ among different treatments in the same genotype according to Duncan's multiple range test.

The transcript levels of EnCOR14a increased $(P<0.05)$ in both genotypes following $6 \mathrm{~h}$ of cold stress and continued to increase for up to $24 \mathrm{~h}$, before decreasing thereafter (Fig. $6 \mathrm{e}, \mathrm{f}$ ). The relative normalized expression of EnCOR14a was significantly higher in melatonin-treated plants compared to untreated plants during the $120 \mathrm{~h}$ of cold stress. A 2.5-fold up-regulation in DX and a 4.6-fold up-regulation in GN were observed in melatonin treated plants after $6 \mathrm{~h}$ of cold treatment. Combination of melatonin and fluridone treatment did not lead to an inhibitory effect in the expression of COR14a compared with melatonin and cold stress treatments. Based on our results, we propose that one of the mechanisms of melatonin may be to protect plant cells from cold injury by regulating cold responsive genes, including CBF transcription factors and cold-responsive genes.

\section{Discussion}

Cold stress is one of the most important abiotic stresses limiting plant growth and causes severe dysfunctions at cellular level. The primary place of cold injury is the cell membrane system ${ }^{38}$. In this study, cold stress resulted in measureable growth restriction and severe oxidative damage in the cold-sensitive GN genotype indicated by increases in electrolyte leakage level, MDA content, and accumulations of $\mathrm{O}_{2}{ }^{-}$and $\mathrm{H}_{2} \mathrm{O}_{2}$. Exogenously applied melatonin alleviated this growth limitation and protected membrane structures against peroxidation during cold stress (Figs 1 and 2). A decline in relative electrolyte leakage and MDA concentration confirmed the protective effect of melatonin in cold stress-induced membrane damage. Melatonin and several of its metabolites are known endogenous free radical scavengers and broad-spectrum antioxidants and have been suggested to directly scavenge $\operatorname{ROS}^{25,28,30}$. Our results revealed that the generation of $\mathrm{H}_{2} \mathrm{O}_{2}$ and $\mathrm{O}_{2}{ }^{\bullet-}$ was inhibited by exogenous melatonin under cold stress. Application of melatonin led to a decline in ROS production, which might be due to direct enhancement of GSH and AsA concentrations as well as CAT, SOD, APX, and GR activities under cold stress (Figs 3 and 4). Pre-treatment with melatonin had a more profound effect on the cold-sensitive genotype compared to the cold-tolerant genotype. These results are consistent with previous study, reporting a primarily antioxidant function of melatonin in plants against abiotic and biotic stresses ${ }^{12}$.

Ample evidence supports the regulatory role of ABA in conferring tolerance to environmental stresses ${ }^{33,39}$. Here, we found that exogenous pre-treatment with ABA efficiently ameliorated cold-induced oxidative damage, characterized by decreased electrolyte leakage, reduced MDA content, and decreased ROS accumulation (Fig. 2). In addition, pre-treatment with ABA enhanced GSH, AsA, total glutathione, and total ascorbate concentrations, 
(a)

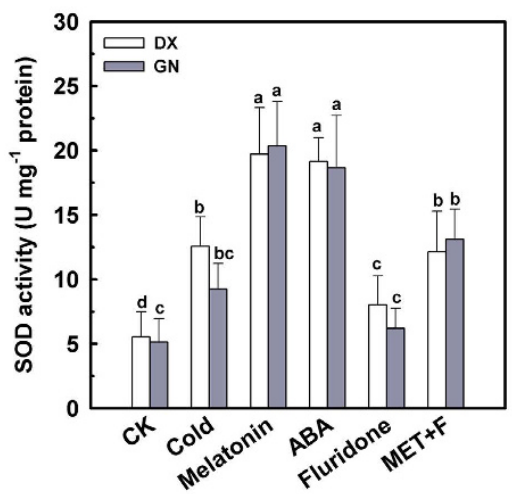

(c)

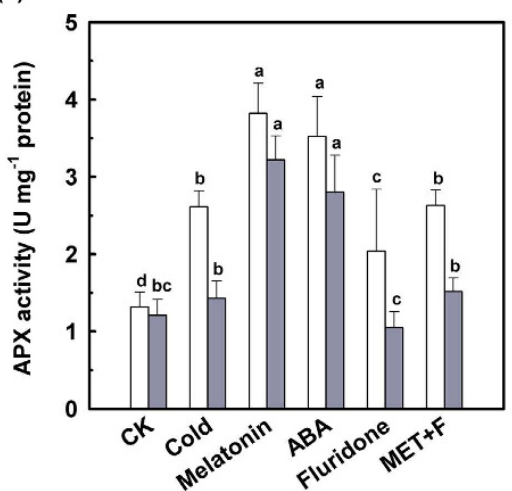

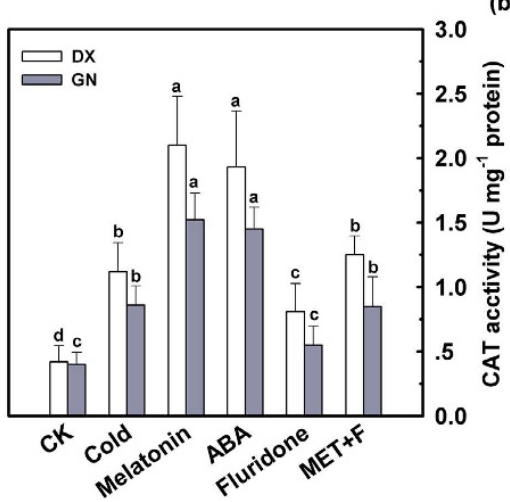

(d)

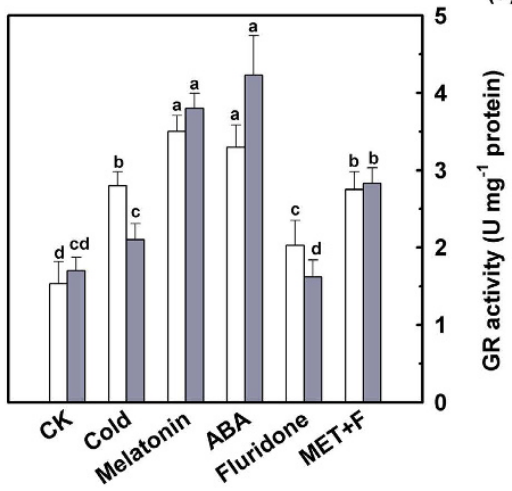

Figure 4. Effect of melatonin and ABA on the activities of antioxidant enzyme SOD (a), CAT (b), APX (c) and GR (d) in leaves of DX and GN under cold stress. The 21-day-old seedlings were pretreated with distilled water, $50 \mu \mathrm{M}$ melatonin, $100 \mu \mathrm{M} \mathrm{ABA}, 50 \mu \mathrm{M}$ fluridone, and $50 \mu \mathrm{M}$ melatonin $+50 \mu \mathrm{M}$ fluridone $(\mathrm{MET}+\mathrm{F})$ for 7 days, respectively, and then exposed to cold stress $\left(4^{\circ} \mathrm{C}\right)$ for $120 \mathrm{~h}$. Plants treated with distilled water under the normal conditions $\left(25^{\circ} \mathrm{C}\right)$ served as controls. Each value represents the mean $\pm \mathrm{SD}$ of three repeats, and different letters above the bars indicate significant differences at $P<0.05$ among different treatments in the same genotype according to Duncan's multiple range test.

as well as SOD, CAT, APX, and GR activities in both genotypes under cold stress. Similar conclusions were observed in pepper under $\mathrm{ABA}$ and low temperature conditions ${ }^{40}$. It has been well documented that $\mathrm{ABA}$ interacted with the important signaling molecules $\mathrm{NO}, \mathrm{H}_{2} \mathrm{O}_{2}$, and $\mathrm{Ca}^{2+}$, participating in the regulation of responses to cold tolerance. However, it is not clear whether ABA is involved in melatonin-induced cold-tolerance in E. nutans. To further investigate the roles of ABA signaling in the response to cold stress and exogenous melatonin treatment, fluridone, an inhibitor of endogenous ABA biosynthesis, was used. When exposed to cold stress, pre-treatments with fluridone in the presence of melatonin inhibited the positive effect of melatonin for cell membranes as indicated by increases in electrolyte leakage, MDA concentration, and ROS levels, and a decline in antioxidant enzymes activities (Figs 3 and 4). These observations suggest that ABA is likely participating in melatonin-induced antioxidant defense under cold stress.

To further investigate the role of melatonin and ABA in the antioxidant defense induced by cold stress, endogenous melatonin and ABA concentrations were assayed. Exposure of E. nutans seedlings to 0-120 h of cold stress gradually and substantially increased endogenous melatonin and ABA concentrations (Fig. 5). However, such increases in endogenous melatonin were unable to ameliorate oxidative stress, which is possibly due to insufficient ROS scavenging by melatonin in parallel with cold-induced ROS generation, especially in cold-sensitive genotype. The higher accumulation of melatonin and ABA in DX compared to GN leaves might contribute to the increased cold tolerance of DX. In agreement with our finding, melatonin-deficient tomatoes were less protected against abiotic stress when compared to the wild type tomato ${ }^{41}$, whereas melatonin-rich plants had an improved ability to withstand such challenges ${ }^{29}$. Accumulation of endogenous melatonin has been suggested to be an adaptive response in plants under cold stress ${ }^{42}$. Treatment with exogenous melatonin significantly increased endogenous melatonin content during cold treatment. As expected, application of melatonin had a more profound effect on cold-sensitive GN than on cold-tolerant DX genotype. It is possible that a mechanism of exogenous melatonin alleviating oxidative injury induced by cold stress, may activate endogenous melatonin synthesis, which in turn acts as an antioxidant and further mediates other defensive pathways for subsequent environmental adaptation. In the present study, an important finding was observed related to melatonin and antioxidant defense induced by cold stress. Melatonin application rapidly enhanced ABA levels in the leaves of both E. nutans genotypes, while $\mathrm{ABA}$ as well as ABA inhibitor applications had no significant effect on endogenous melatonin production (Fig. 5). 
(a)

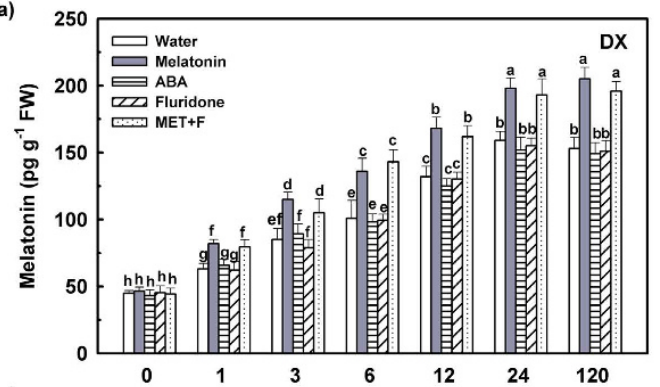

(c)

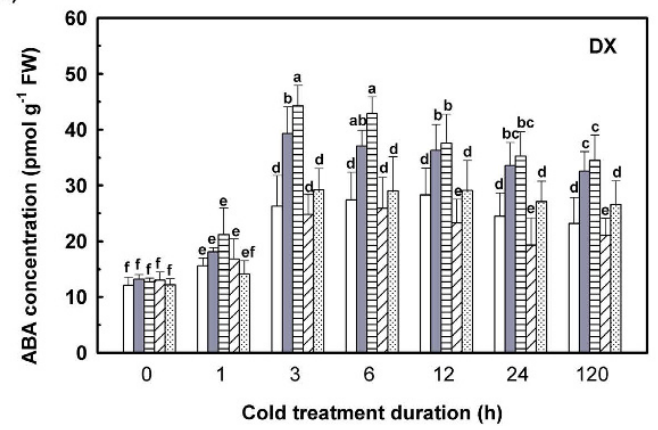

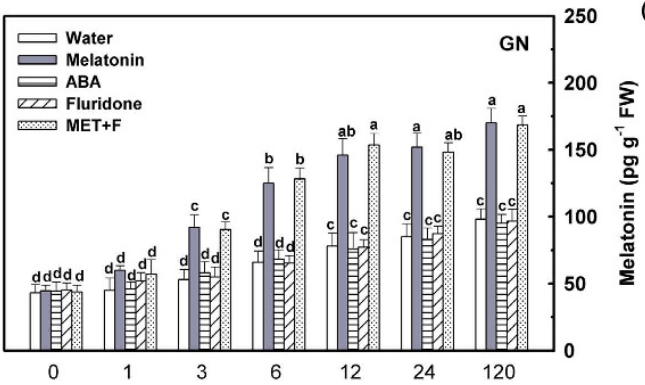

(d)

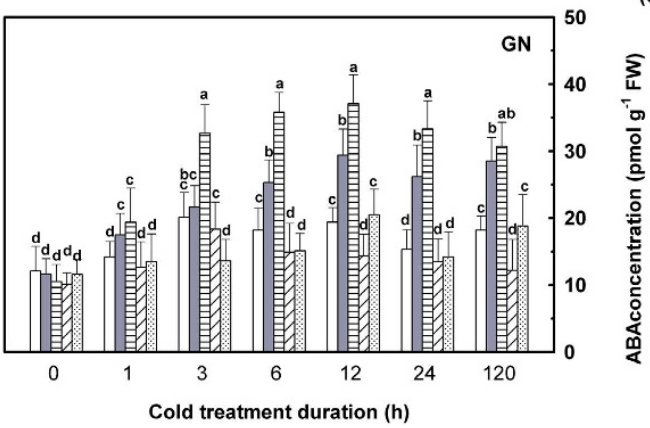

Figure 5. Endogenous melatonin (a) and ABA (b) concentration in leaves of DX and GN under cold stress. The 21-day-old seedlings were pretreated with distilled water, $50 \mu \mathrm{M}$ melatonin, $100 \mu \mathrm{M} \mathrm{ABA}, 50 \mu \mathrm{M}$ fluridone, and $50 \mu \mathrm{M}$ melatonin $+50 \mu \mathrm{M}$ fluridone $(\mathrm{MET}+\mathrm{F})$ for 7 days, respectively, and then exposed to cold stress $\left(4^{\circ} \mathrm{C}\right)$. Samples were taken at $0,1,3,6,12,24$, and $120 \mathrm{~h}$ of cold treatment. Untreated controls were taken at time zero after the experiment began. Each value represents the mean of three replicates $\pm S D$, and different letters above the bars indicate significant differences at $P<0.05$ among different time points in the same genotype according to Duncan's multiple range test.

These observations demonstrated that ABA might act as a downstream signal of melatonin in the antioxidant defense responses of plants.

Recent studies have reported that melatonin plays central roles in the regulation of gene expression and antioxidant activities ${ }^{30}$. Several important cold-related genes were selected. The present result indicated that exogenous melatonin up-regulated components of the cold-stress signaling pathways following specific time intervals of cold exposure. Transcripts of EnCBF9 and EnCBF14 accumulated immediately after 1 and $3 \mathrm{~h}$ of cold treatment. The induction of $C B F s$ in plants up-regulated the expression of COR genes such as COR14b, leading to increased freezing tolerance ${ }^{10}$. Cold stress induced higher EnCOR14a expression in the cold-tolerant genotypes DX compared to the cold-sensitive genotype GN (Fig. 6), thus supporting the findings of Ndong et al. ${ }^{11}$ who pointed out that group 3 late embryogenesis abundant proteins were induced in cold-treated wheat and rye plants. The increased expression of EnCBF9, EnCBF14, and EnCOR14a was induced by melatonin treatment, revealed by a comparison with untreated plants under cold stress. These observations indicate that melatonin might serve as a second messenger, activating downstream cold-responsive genes including EnCBF9, EnCBF14, and $E n C O R 14 a$, and thus stimulating biosynthesis of cold-protecting compounds. Consequently, this contributes to the enhancement of cold tolerance in plants treated with exogenous melatonin and subjected to cold stress ${ }^{23,31}$. Previous studies reported that both cold treatment and exogenous ABA treatment induce the expression of CBF1 and several $C O R$ genes in $A$. thaliana plants ${ }^{43,44}$. In contrast, our study revealed that application of exogenous $\mathrm{ABA}$ and fluridone failed to further change expressions of EnCBFs and EnCOR14a genes under cold stress. This however, is in accordance with the findings of Skinner et al. ${ }^{45}$ who found that ABA-treated barley under cold stress did not affect $C B F$ gene expression. The results suggested that activation of $C B F$ and $C O R$ gene expression can regulated through both ABA-dependent and ABA-independent pathways ${ }^{5,46}$.

\section{Conclusions}

We found that both exogenous melatonin and ABA have the ability to alleviate cold stress induced oxidative damage. Exogenous melatonin improved cold tolerance via induction of endogenous melatonin production, which might serve as a second messenger activating downstream cold-responsive genes such as EnCBF9, EnCBF14, and $E n C O R 14 a$, thus stimulating antioxidant defense systems alleviating ROS accumulation-induced oxidative damage. Further investigations revealed that this melatonin-induced antioxidant defense may function via ABA-dependent and ABA-independent signaling pathways. However, the molecular network that operates during cold stress and that is mediated by melatonin remains to be determined. Further studies are necessary to elucidate the interrelation between melatonin and other signaling molecules in response to cold stress. 
(a)

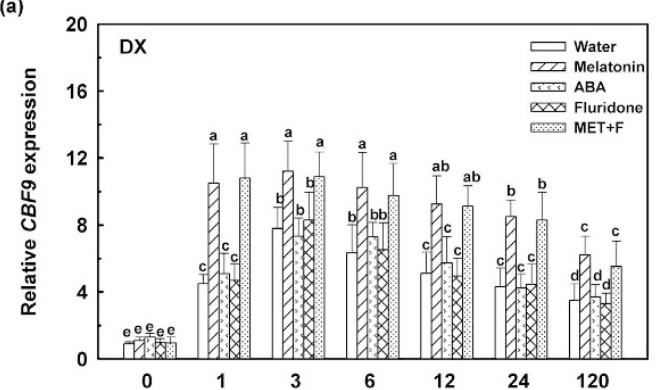

(c)

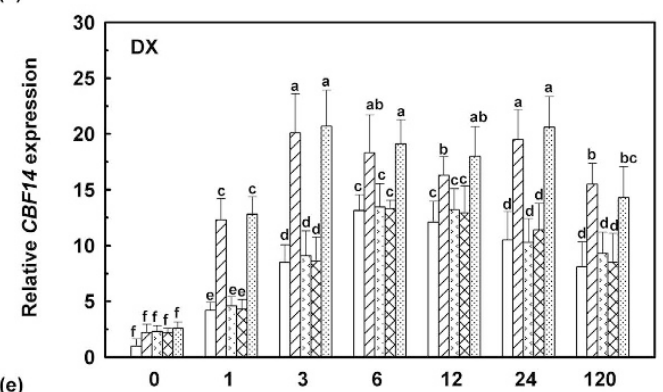

(e)

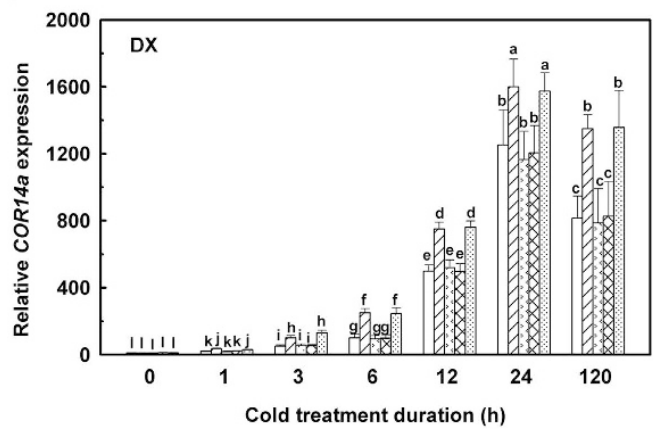

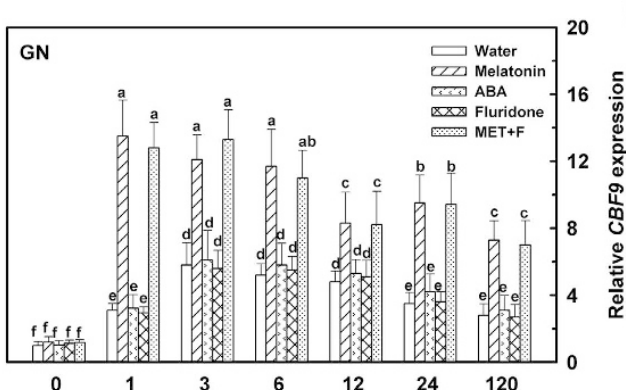

(b)

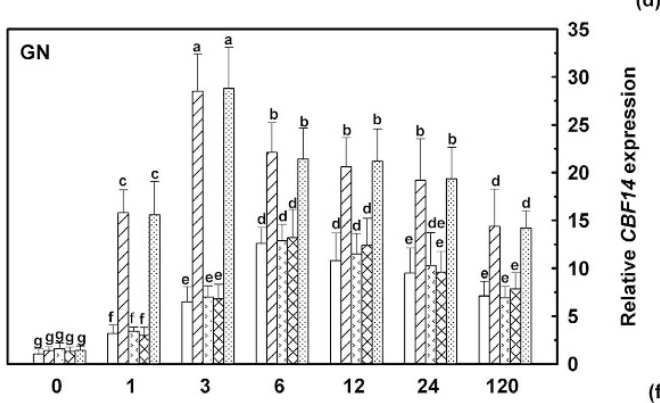

(f)

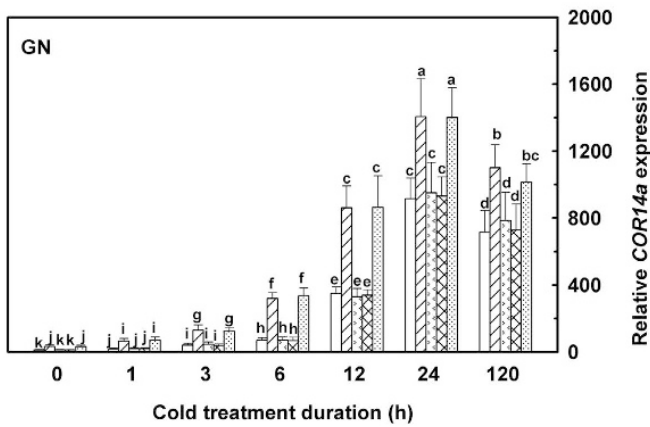

Figure 6. Expression of cold-related genes in leaves of DX and GN under cold stress. The 21-day-old seedlings were pretreated with distilled water, $50 \mu \mathrm{M}$ melatonin, $100 \mu \mathrm{M} \mathrm{ABA}, 50 \mu \mathrm{M}$ fluridone, and $50 \mu \mathrm{M}$ melatonin $+50 \mu \mathrm{M}$ fluridone $(\mathrm{MET}+\mathrm{F})$ for 7 days, respectively, and then exposed to cold stress $\left(4^{\circ} \mathrm{C}\right)$. Samples were taken at $0,1,3,6,12,24$, and $120 \mathrm{~h}$ of cold treatment. Untreated controls were taken at time zero after the experiment began. Each value represents the mean of three replicates $\pm S D$, and different letters above the bars indicate significant differences at $P<0.05$ among different time points in the same genotype according to Duncan's multiple range test.

\section{Materials and Methods}

Plant materials and experimental design. Elymus nutans Griseb. seeds were obtained from two sources: seeds of Damxung (DX) were collected in September 2015, from wild plants growing in Damxung County $\left(30^{\circ} 28.535^{\prime} \mathrm{N}, 91^{\circ} 06.246^{\prime} \mathrm{E}\right.$, altitude $\left.4678 \mathrm{~m}\right)$, located in the middle of Tibet, China. And Gannan (GN) seeds were obtained from Lanzhou Xinglong Grass Industry Technology Service CO. Ltd., China in September 2015. The seeds were cleaned and stored at $4^{\circ} \mathrm{C}$ in paper bags until the start of the experiments.

DX and GN seeds were surface-sterilized in $0.1 \%(\mathrm{w} / \mathrm{v})$ sodium hypochlorite, and germinated on moistened filter paper for 7 days at $25^{\circ} \mathrm{C}$. Morphologically uniform seedlings were transferred to plates using a 1: 1 (v/v) mixture of vermiculite and sand as solid support. Plants were germinated and grown in a growth chamber at a day/night temperature $25 / 25^{\circ} \mathrm{C}$, a relative humidity of $70 / 60 \%$, a day/night regime of $14 / 10 \mathrm{~h}$ and a photosynthetic photon flux density (PPFD) of $300 \mu \mathrm{mol} \mathrm{m} \mathrm{m}^{-2} \mathrm{~s}^{-1}$. Light was provided by a fluorescent lamp (Philips Electronics N. V. Holland, Nanjing, China). To investigate the role of exogenous melatonin and ABA in plant physiological responses and cold stress resistance, 21-day-old $E$. nutans seedlings were irrigated with water or with different concentrations of melatonin $(0,1,10,50,100,300 \mu \mathrm{M}), \mathrm{ABA}(0,5,10,50,100,200 \mu \mathrm{M})$, and $50 \mu \mathrm{M}$ fluridone (ABA biosynthesis inhibitor) for 7 days, respectively ${ }^{31}$. After 7 days of pretreatment, the 28-day-old plants were subjected to cold stress $\left(4^{\circ} \mathrm{C}\right)$ for $120 \mathrm{~h}$. Samples were taken at $0,1,3,6,12,24$, and $120 \mathrm{~h}$ of cold treatment, immediately flash frozen in liquid nitrogen and stored at $-80^{\circ} \mathrm{C}$ until analyzed. After $120 \mathrm{~h}$ of cold treatment, fresh weight of shoots, cell membrane response, ROS accumulation, non-enzymatic antioxidants concentration and related antioxidant enzyme activities were measured. For expression of cold-related genes, endogenous melatonin, and $\mathrm{ABA}$ content were quantification at different time intervals of cold stress. Untreated 


\begin{tabular}{|l|l|}
\hline Primer Name & Primer Sequences $\left(\mathbf{5}^{\prime}-\mathbf{3}^{\prime}\right)$ \\
\hline EnCBF9-F & GGAGCTGCTTGTTCGACTAAT \\
\hline EnCBF9-R & AACCGGGAACTCGACAGATA \\
\hline EnCBF14-F & GAGGCTCTCACTATGAACGAAC \\
\hline EnCBF14-R & TGGATGGTTTCTCTGTTTCTGT \\
\hline EnCOR14a-F & CGATCAGCACGGAAGAAGAA \\
\hline EnCOR14a-R & GCAAGTGCAAATTAGCTCATACA \\
\hline En18s $r R N A-\mathrm{F}$ & GCCTCGTTTCCTGCTCTTAT \\
\hline En18s $r R N A-\mathrm{R}$ & CCGCTCCAGAACAACATCT \\
\hline
\end{tabular}

Table 1. Primer sequences used for qRT-PCR.

controls were taken at time zero after the experiment began. All assessments were conducted in three biological replicates. Three replicates from independent plates (each consisting of 15 plants) were harvested for each treatment.

Determination of plant growth characters. Three healthy seedlings were randomly chosen from each group after $120 \mathrm{~h}$ of cold stress. The shoots of the seedlings were cut at the growth medium line and their fresh weights were recorded.

Assay of electrolyte leakage and malondialdehyde content. Electrolyte leakage was determined according to the method of Song et al. ${ }^{47}$ with some modifications. The fresh leaves $(0.5 \mathrm{~g})$ were washed in deionized water and placed in Petri dishes with $5 \mathrm{ml}$ deionized water at $25^{\circ} \mathrm{C}$ for $2 \mathrm{~h}$. After the incubation, the conductivity was measured. Then, the samples were boiled for $20 \mathrm{~min}$ and conductivity was read again. The electrolyte leakage was expressed as percent.

Malondialdehyde (MDA) content was extracted using chilled thiobarbituric acid (TBA) reagent according to the method of Dhindsa et al. ${ }^{48}$. The absorbance of the supernatant was measured at 450,532 , and $600 \mathrm{~nm}$.

Quantification of ROS Accumulation. The concentration of $\mathrm{H}_{2} \mathrm{O}_{2}$ was measured by monitoring the absorbance of titanium-peroxide complex at $415 \mathrm{~nm}$ according to the method described by Shi et al. ${ }^{49}$.

Superoxide radical production rate was determined by the plant $\mathrm{O}_{2}{ }^{--}$ELISA Kit (DG, Beijing, China) based on antibody-antigen-enzyme-antibody complex following the manufacturer's instruction.

Measurement of Non-enzymatic Antioxidant Concentrations and Antioxidant Enzyme Activities.

A leaf sample $(0.4 \mathrm{~g})$ was ground with a mortar and pestle in $2 \mathrm{ml}$ of $0.5 \mathrm{M}$ EDTA solution containing $3 \%$ trichloroacetic acid and centrifuged at $15,000 \times g$ for $10 \mathrm{~min}$ at $4{ }^{\circ} \mathrm{C}$. The supernatant was used for assays of the levels of ascorbic acid (AsA) and glutathione (GSH). The amount of GSH was evaluated following the method of Anderson $^{50}$ and was expressed as $\mu \mathrm{g} \mathrm{g}^{-1} \mathrm{FW}$. The amount of AsA was estimated using the method of Foyer and Halliwei ${ }^{51}$ and was expressed as $\mu \mathrm{g} \mathrm{g}^{-1} \mathrm{FW}$.

The leaves $(0.5 \mathrm{~g})$ were homogenized with a mortar and pestle at $4^{\circ} \mathrm{C}$ in $5 \mathrm{ml} 50 \mathrm{mM}$ phosphate buffer $(\mathrm{pH}$ 7.8) containing $1 \mathrm{mM}$ EDTA and $2 \%$ PVP. Homogenate was centrifuged at $12,000 \times g$ for $20 \mathrm{~min}$ at $4{ }^{\circ} \mathrm{C}$ and the supernatant was used for enzyme activity assays. Protein content in the supernatant was determined according to Bradford ${ }^{52}$.

The assay for ascorbate peroxidase (APX) activity was measured in a reaction mixture of $3 \mathrm{ml}$ containing $100 \mathrm{mM}$ phosphate ( $\mathrm{pH} 7$ ), $0.1 \mathrm{mM}$ EDTA- $\mathrm{Na}_{2}, 0.3 \mathrm{mM}$ ascorbic acid, $0.06 \mathrm{mM} \mathrm{H}_{2} \mathrm{O}_{2}$ and $100 \mu \mathrm{l}$ enzyme extract. Change in absorption was observed at $290 \mathrm{~nm} 30 \mathrm{~s}$ after addition of $\mathrm{H}_{2} \mathrm{O}_{2}{ }^{53}$. One unit of APX forms $1 \mu \mathrm{M}$ of ascorbate oxidized per minute under assay conditions. The activity of catalase (CAT) was measured by following the consumption of $\mathrm{H}_{2} \mathrm{O}_{2}$ at $240 \mathrm{~nm}$ according to Cakmak and Marschner ${ }^{54}$. The decrease in the absorption was followed for $3 \mathrm{~min}$ and a breakdown of $1.0 \mu \mathrm{M} \mathrm{H}_{2} \mathrm{O}_{2} \mathrm{ml}^{-1} \mathrm{~min}^{-1}$ was defined as 1 Unit of CAT activity. Glutathione reductase (GR) activity was measured by following the decrease in absorbance at $340 \mathrm{~nm}$ due to NADPH oxidation. The reaction mixture contained tissue extract, $1 \mathrm{mM}$ EDTA, $0.5 \mathrm{mM}$ GSSG, $0.15 \mathrm{mM}$ NADPH and $50 \mathrm{mM}$ Tris- $\mathrm{HCl}$ buffer ( $\mathrm{pH} 7.5$ ) and $3 \mathrm{mM} \mathrm{MgCl}_{2}{ }^{55}$. The reaction was started by adding NADPH. Activity of superoxide dismutase (SOD) was determined according to Beauchamp and Fridovich ${ }^{56}$ by following the photo-reduction of nitroblue tetrazolium (NBT) at $560 \mathrm{~nm}$.

Quantification of Endogenous Melatonin by Enzyme-linked Immunosorbent Assay. Melatonin was extracted using the acetone-methanol method as previously described ${ }^{57}$. The supernatant was transfered to a new centrifuge tube containing $0.5 \mathrm{ml}$ of $1 \%$ trichloric acid for protein precipitation. After centrifugation at $12,000 \times \mathrm{g}$ for $10 \mathrm{~min}$ at $4{ }^{\circ} \mathrm{C}$, the extract was used for quantification of melatonin using the Plant Melatonin ELISA Kit (RD, USA).

Determination of ABA Content. The quantitative determination of ABA in leaves of DX and GN plants were carried out using enzyme-linked immunosorbent assay (RD, USA). Extraction and purification prior to immunoassay have been described by Zhang et al. ${ }^{58}$.

Extraction of Total RNA and Quantitative Real-time PCR (qRT-PCR) Analyses. Total RNA was isolated from leaves using RNAiso Reagent (TaKaRa, Dalian, China). Gel electrophoresis was performed and 
absorbance measured at 260 and $280 \mathrm{~nm}$ to ensure RNA integrity. RNA was reverse-transcribed with PrimeScript RT reagent Kit with gDNA Eraser (Takara, Dalian, China). Synthesized cDNA was subjected to Polymerase chain reaction (PCR) for 40 cycles using primers as described in Table 1. The PCR primers were designed using Primer Premier 5 (Version 5.0 for Windows and Power Macintosh, Palo Alto, CA). Each gene was obtained from our previous transcriptome data of E. nutans (accession No: SRP074469). The PCR conditions consisted of denaturation at $95^{\circ} \mathrm{C}$ for $3 \mathrm{~min}$, followed by 40 cycles of denaturation at $95^{\circ} \mathrm{C}$ for $30 \mathrm{~s}$, annealing at $60^{\circ} \mathrm{C}$ for $30 \mathrm{~s}$, and extension at $72^{\circ} \mathrm{C}$ for $30 \mathrm{~s}$. The relative expression levels of target gene were calculated with formula $2^{-\Delta \Delta \mathrm{CT}}$ method $^{59}$.

Statistical Analysis. Each experiment was repeated three times. All values were expressed as means \pm SD. The data were analyzed via one-way ANOVA using SPSS-17 statistical software (SPSS Inc., Chicago, IL, USA), followed by Duncan's tests. A $p$-value of $<0.05$ indicated a significant difference.

\section{References}

1. Chinnusamy, V., Zhu, J. K. \& Sunkar, R. Gene regulation during cold stress acclimation in plants In: Sunkar, R. eds. Plant Stress Tolerance. Springer, Heidelberg 39-55 (2010).

2. Mittler, R. Oxidative stress, antioxidants and stress tolerance. Trends Plant Sci. 7, 405-410 (2002).

3. Beck, E. H., Heim, R. \& Hansen, J. Plant resistance to cold stress: mechanisms and environmental signals triggering frost hardening and dehardening. J. Biosci. 29, 449-459 (2004).

4. Thomashow, M. F. Plant cold acclimation: Freezing tolerance genes and regulatory mechanisms. Annu. Rev. Plant Physiol. Plant Mol. Biol. 50, 571-599 (1999).

5. Shinozaki, K. \& Yamaguchi-Shinozaki, K. Molecular responses to dehydration and low temperature: Differences and cross-talk between two stress signaling pathways. Curr. Opin. Plant Biol. 3, 217-223 (2000).

6. Chinnusamy, V. et al. ICE1: A regulator of cold-induced transcriptome and freezing tolerance in Arabidopsis. Gene Dev. 17, 1043-1054 (2003).

7. Vágújfalvi, A. et al. The expression of several Cbf genes at the Fr-A2 locus is linked to frost resistance in wheat. Molecul. Genet. Genomics 274, 506-514 (2005).

8. Knox, A. K. et al. Identification of candidate CBF genes for the frost tolerance locus Fr-Am2 In Triticum monococcum. Plant Mol. Biol. 667, 257-270 (2008).

9. Fricano, A. et al. Genetic variants of $\mathrm{HvCbf14}$ are statistically associated with frost tolerance in a European germplasm collection of Hordeum vulgare. Theor. Appl. Genet. (TAG) 119, 1335-1348 (2009).

10. Vashegyi, I. et al. Cold response of dedifferentiated barley cells at the gene eexpression, hormone composition, and freezing tolerance levels: studies on callus cultures. Mol. Biotechnol. 54, 337-349 (2013).

11. Ndong, C. N. et al. Cold-regulated cereal chloroplast late embryogenesis abundant-like proteins. Molecular characterization and functional analyses. Plant Physiol. 129, 1368-1381 (2002).

12. Leung, J. \& Giraudat, J. Abscisic acid signal transduction. Annu. Rev. Plant Physiol. 49, 199-222 (1998).

13. Chinnusamy, V., Schumaker, K. \& Zhu, J. K. Molecular genetic perspectives on cross-talk and specificity in abiotic stress signalling in plants. J. Exp. Bot. 55, 225-236 (2004).

14. Roychoudhury, A., Paul, S. \& Basu, S. Cross-talk between abscisic acid-dependent and abscisic acid-independent pathways during abiotic stress. Plant Cell Rep. 32, 985-1006 (2013).

15. Peleg, Z. \& Blumwald, E. Hormone balance and abiotic stress tolerance in crop plants. Curr. Opin. Plant Biol. 14, 290-295 (2011).

16. Calvo, J. R., Gonzalez-Yanes, C. \& Maldonado, M. D. The role of melatonin in the cells of the innate immunity: a review. J. Pineal. Res. 55, 103-120 (2013).

17. Tan, D. X. et al. Functional roles of melatonin in plants, and perspectives in nutritional and agricultural science. J. Exp. Bot. 63, 577-597 (2012)

18. Zhang, N. et al. The RNA-seq approach to discriminate gene expression profiles in response to melatonin on cucumber lateral root formation. J. Pineal. Res. 56, 39-50 (2014).

19. Byeon, Y. et al. Light regulated melatonin biosynthesis in rice during the senescence process in detached leaves. J. Pineal. Res. 53, 107-111 (2012)

20. Shi, H. et al. Comparative physiological, metabolomic, and transcriptomic analyses reveal mechanisms of improved abiotic stress resistance in bermudagrass [Cynodon dactylon (L). Pers.] by exogenous melatonin. J. Exp. Bot. 66, 681-694 (2015).

21. Kang, K., Lee, K., Park, S., Kim, Y. S. \& Back, K. Enhanced production of melatonin by ectopic overexpression of human serotonin $\mathrm{N}$-acetyltransferase plays a role in cold resistance in transgenic rice seedlings. J. Pineal. Res. 49, 176-182 (2010).

22. Lei, X. Y., Zhu, R. Y., Zhang, G. Y. \& Dai, Y. R. Attenuation of cold-induced apoptosis by exogenous melatonin in carrot suspension cells: the possible involvement of polyamines. J. Pineal. Res. 36, 126-131 (2004).

23. Turk, H. \& Erdal, S. Melatonin alleviates cold-induced oxidative damage in maize seedlings by up-regulating mineral elements and enhancing antioxidant activity. J. Plant Nutr. Soil Sci. 178, 433-439 (2015).

24. Zhang, H. J. et al. Melatonin promotes seed germination under high salinity by regulating antioxidant systems, ABA and GA4 interaction in cucumber (Cucumis sativus L.). J. Pineal. Res. 57, 269-279 (2014).

25. Hasan, M. K. et al. Melatonin mitigates cadmium phytotoxicity through modulation of phytochelatins biosynthesis, vacuolar sequestration, and antioxidant potential in Solanumly copersicum L. Front. Plant Sci. 6, 601 (2015).

26. Yin, L. et al. Exogenous melatonin improves Malus resistance to Marssonina apple blotch. J. Pineal. Res. 54, 426-439 (2013).

27. Zhang, N. et al. Roles of melatonin in abiotic stress resistance in plants. J. Exp. Bot. 66, 647-656 (2015).

28. Posmyk, M., Bałabusta, M., Wieczorek, M., Sliwinska, E. \& Janas, K. M. Melatonin applied to cucumber (Cucumis sativus L.) seeds improves germination during chilling stress. J. Pineal. Res. 46, 214-223 (2009).

29. Park, S. et al. Melatoninrich transgenic rice plants exhibit resistance to herbicide-induced oxidative stress. J. Pineal. Res. 54, 258-263 (2013).

30. Bajwa, V. S., Shukla, M. R., Sherif, S. M., Murch, S. J. \& Saxena, P. K. Role of melatonin in alleviating cold stress in Arabidopsis thaliana. J. Pineal. Res. 56, 238-245 (2014).

31. Shi, H. \& Chan, Z. The cysteine2/histidine2-type transcription factor ZINC FINGER OFARABIDOPSIS THALIANA 6-activated C-REPEATBINDING FACTOR pathway is essential for melatonin-mediated freezing stress resistance In Arabidopsis. J. Pineal. Res. 57, 185-191 (2014).

32. Kosová, K. et al. Complex phytohormone responses during the cold acclimation of two wheat cultivars differing in cold tolerance, winter Samanta and spring Sandra. J. Plant Physiol. 169, 567-576 (2012).

33. Karimi, R. \& Ershadi, A. Role of exogenous abscisic acid in adapting of 'Sultana' grapevine to low-temperature stress. Acta Physiol. Plant. 37, 151 (2015).

34. Neill, S. et al. Nitric oxide, stomatal closure, and abiotic stress. J. Exp. Bot. 59, 165-176 (2008).

35. Guo, Z. F. et al. Abscisic acid, $\mathrm{H}_{2} \mathrm{O}_{2}$ and nitric oxide interactions mediated cold-induced S-adenosylmethionine synthetase in Medicago sativa subsp. falcata that confers cold tolerance through up-regulating polyamine oxidation. Plant Biotech. J. 12, 601-612 (2014). 
36. Ma, F. F. et al. Nitric oxide-activated calcium/calmodulin-dependent protein kinase regulates the abscisic acid-induced antioxidant defence in maize. J. Exp. Bot. 63, 4835-4847 (2012).

37. Wang, W. Y., Wang, Q. J. \& Wang, H. C. The effect of land management on plant community composition, species diversity, and productivity of alpine Kobersia steppe meadow. Ecol. Res. 21, 181-187 (2006).

38. Lyons, J. M. Chilling injury in plants. Annu. Rev. Plant Physiol. 24, 445-466 (1973).

39. Fujita, Y. et al. AREB1 is a transcription activator of novel ABRE-dependent ABA signaling that enhances drought stress tolerance in Arabidopsis. Plant Cell 17, 3470-3488 (2005).

40. Guo, W. L. et al. Exogenous abscisic acid increases antioxidant enzymes and related gene expression in pepper (Capsicum annuum) leaves subjected to chilling stress. Genet. Mol. Res. 11, 4063-4080 (2012).

41. Okazaki, M., Higuchi, K., Aouini, A. \& Ezura, H. Lowering intercellular melatonin levels by transgenic analysis of indoleamine 2, 3-dioxygenase from rice in tomato plants. J. Pineal. Res. 49, 239-247 (2010).

42. Byeon, Y. et al. Coordinated regulation of melatonin synthesis and degradation genes in rice leaves in response to cadmium treatment. J. Pineal. Res. 58, 470-478 (2015).

43. Knight, H., Zarka, D. G., Okamoto, H., Thomashow, M. F. \& Knight, M. R. Abscisic acid induces CBF gene transcription and subsequent induction of cold-regulated genes via the CRT promoter element. Plant Physiol. 135, 1710-1717 (2004).

44. Gusta, L. V., Trischuk, R. \& Weiser, C. J. Plant cold acclimation: the role of abscisic acid. J. Plant Growth Regul. 24, 308-318 (2005).

45. Skinner, J. S. et al. Structural, functional, and phylogenetic characterization of a large CBF gene family in barley. Plant Mol. Biol. 59, 533-551 (2005).

46. Kurepin, L. V. et al. Role of CBFs as integrators of chloroplast redox, phytochrome and plant hormone signaling during cold acclimation. Int. J. Mol. Sci. 14, 12729-12763 (2013).

47. Song, L., Ding, W., Zhao, M., Sun, B. \& Zhang, L. Nitric oxide protects against oxidative stress under heat stress in the calluses from two ecotypes of reed. Plant Sci. 171, 449-458 (2006).

48. Dhindsa, R. S., Plumb-dhindsa, P. \& Thorpe, T. A. Leaf senescence: correlated with increased levels of membrane permeability and lipid peroxidation and decreased levels of superoxide dismutase and catalase. J. Exp. Bot. 32, 93-101 (1981).

49. Shi, H. et al. Manipulation of arginase expression modulate abiotic stress tolerance in Arabidopsis: effect on arginine metabolism and ROS accumulation. J. Exp. Bot. 64, 1367-1379 (2013).

50. Anderson, M. E. Determination of glutathione and glutathione disulfide in biological samples. Methods Enzymol. 113, 548-555 (1985).

51. Foyer, C. H. \& Halliwei, I. B. The presence of glutathione and glutathione reductase in chloroplasts: a proposed role in ascorbic acid metabolism. Planta 133, 21-25 (1976).

52. Bradford, M. M. A rapid and sensitive method for the quantitation of microgram quantities of protein utilizing the principle of protein-dye binding. Anal. Biochem. 72, 248-254 (1976).

53. Nakano, Y. \& Asada, K. Hydrogen peroxide is scavenged by ascorbate-specific peroxidase in spinach chloroplast. Plant Cell Physiol. 22, 867-880 (1981)

54. Cakmak, I. \& Marschner, H. Magnesium deficiency and high light intensity enhance activities of superoxide dismutase, ascorbate peroxidase, and glutathione reductase in bean leaves. Plant Physiol. 98, 1222-1227 (1992).

55. Shaedle, M. \& Bassham, J. A. Chloroplast glutathione reductase. Plant Physiol. 59, 1011-1012 (1977).

56. Beauchamp, C. \& Fridovich, I. Superoxide dismutase: Improved assays and an assay applicable to acrylamide gels. Anal. Biochem. 44, 276-287 (1971)

57. Pape, C. \& Lüning, K. Quantification of melatonin in phototrophic organisms. J. Pineal. Res. 41, 157-165 (2006).

58. Zhang, F. J., Jin, Y. J., Xu, X. Y., Lu, R. C. \& Chen, H. J. Study on the extraction, purification and quantification of jasmonic acid, abscisic acid and indole-3-acetic acid in plants. Phytochem. Analysis 19, 560-567 (2008).

59. Livak, K. J. \& Schmittgen, T. D. Analysis of relative gene expression data using real-time quantitative PCR and the 2(-Delta Delta C(T) method. Methods 25, 402-408 (2001).

\section{Acknowledgements}

This work was supported by the National Natural Science Foundation of China (No. 31402129), National Natural Science Foundation of China (No. 31272490), and Natural Science Foundation of Shaanxi Province (No. 2016JM3025).

\section{Author Contributions}

J.F., T.H. and Y.X. designed the research; Y.W., E.Z., J.W., H.S., Q.L. and Y.X. performed the experiment; J.F. analyzed the data and wrote the article; Y.M. and Y.X. provided the experimental materials. All authors read and approved the manuscript.

\section{Additional Information}

Supplementary information accompanies this paper at http://www.nature.com/srep

Competing financial interests: The authors declare no competing financial interests.

How to cite this article: Fu, J. et al. Improved cold tolerance in Elymus nutans by exogenous application of melatonin may involve ABA-dependent and ABA-independent pathways. Sci. Rep. 7, 39865; doi: 10.1038/ srep39865 (2017).

Publisher's note: Springer Nature remains neutral with regard to jurisdictional claims in published maps and institutional affiliations.

(c) (i) This work is licensed under a Creative Commons Attribution 4.0 International License. The images or other third party material in this article are included in the article's Creative Commons license, unless indicated otherwise in the credit line; if the material is not included under the Creative Commons license, users will need to obtain permission from the license holder to reproduce the material. To view a copy of this license, visit http://creativecommons.org/licenses/by/4.0/

(c) The Author(s) 2017 